\title{
LA MORFOLOGÍA DEL TEMA DE AORISTO EN LOS LÉXICOS Y GRAMÁTICOS ATICISTAS (SIGLOS I-III D.C.)
}

\author{
MARÍA BELÉN BONED \\ Universidad Complutense de Madrid \\ mabelbon@ucm.es \\ ORCID: 0000-0001-7917-0288
}

\section{RESUMEN}

En los siglos I-III d.C. la lengua griega ya ha sufrido notables cambios a nivel fonético, morfológico y sintáctico respecto al griego ático del siglo V a.C. Por esa razón, hacia el siglo II d.C. surgen los llamados gramáticos aticistas, que tienen como objetivo corregir los cambios que está sufriendo el griego teniendo como modelo el dialecto ático. En este breve estudio, se pretende describir la situación morfológica del aoristo en los SIGLOS I-III d.C. a partir de las denuncias que hacen los gramáticos aticistas sobre las formas de aoristo que consideran incorrectas y verificar en qué medida estas denuncias se reflejan en la literatura de estos siglos.

PALABRAS CLAVE: morfología, aoristo, aticismo, diacronía, koiné.

\section{MORPHOLOGY OF THE AORIST'S THEME IN THE ATTICIST LEXICA AND GRAMMARS (I-III AD)}

\section{ABSTRACT}

In the 2nd-3rd centuries AD the Greek language had already undergone considerable phonetic, morphological and syntax changes from the Attic Greek of the 5th century BC. Therefore, ca. 2nd century AD the so-called Atticist Grammarians appeared, whose objective was to correct the undergoing changes of the Greek language using the attic dialect as a model. This brief study aims to describe the morphological situation of the aorist during the 1st-3rd centuries AD examining the complaints of the Atticist Grammarians towards the forms of the aorist which they considered to be incorrect, as well as to verify in what measure such complaints were reflected on the literature of those centuries.

KEYWORDS: morphology, aorist, Atticism, diachrony, koiné.

\section{INTRODUCCIÓN}

Para la realización de este estudio sobre la morfología del tema de aoristo en los siglos I-III d.C., se han utilizado las ediciones presentes en el Thesaurus Linguae Graecae $(T L G)^{1}$ de los gramáticos aticistas Frínico, Meris, Herodiano, Oro, Elio Dionisio, Timeo y el Antiaticista (quien busca ejemplos de lo que los gramáticos anteriores condenan en la literatura de época clásica). Se han utilizado las glosas en las que denuncian formas de aoristo que consideran de nueva creación e

${ }^{1} T L G=$ Thesaurus Linguae Graecae Digital Library: <http://www.tlg.uci.edu> (acceso: 20 de mayo de 2020). 
incorrectas respecto al ático del siglo V a.C., aunque, como veremos, en algunas ocasiones se equivocan. ${ }^{2}$ Todos ellos vivieron y escribieron entre los siglos II-IV d.C., época en la que la lengua griega empieza a tener cada vez más rasgos que la acercan al griego moderno y la alejan del antiguo dialecto ático que tanto buscan imitar estos gramáticos aticistas. ${ }^{3}$

Las glosas, para aquellos que no las conozcan, tienen todas la misma estructura: primero, las formas de aoristo que tratan y, después, las formas que consideran adecuadas (1a). En ocasiones incluyen una pequeña explicación sobre los cambios que se han producido (1b) o una cita de algún autor clásico mostrando el uso ático (1c).

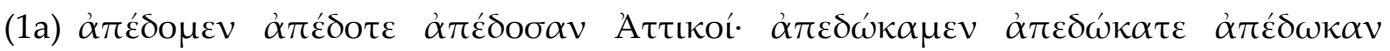
"E $\lambda \lambda \eta v \varepsilon \varsigma$. (Moer. $\alpha$ 19)

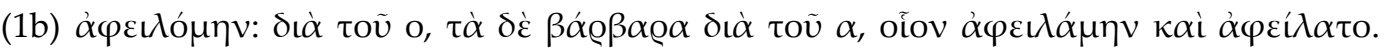

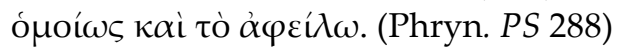

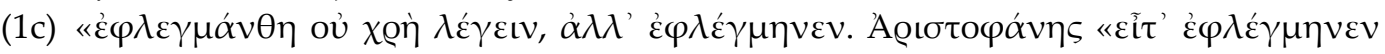

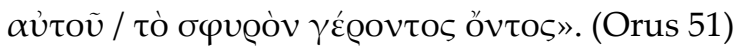

A la hora de llevar a cabo este estudio, por un lado, se han agrupado las glosas de los gramáticos por raíces (treinta y cuatro en total) con lo cual se analizan juntas en un solo grupo las glosas que denuncian formas de aoristo de un mismo verbo. A su vez, los grupos de glosas están clasificados en: a) aoristos radicales atemáticos; $b$ ) aoristos intransitivos en $-\eta-y-\theta \eta-; c)$ aoristos radicales temáticos; y d) aoristos sigmáticos.

Por otro lado, la búsqueda se ha limitado al corpus literario del TLG, acotándolo a los autores y obras de los siglos I-III d.C., y concretamente a las formas de aoristo que mencionan los gramáticos para verificar en qué medida aparecen en los textos. De este modo, si condenan, por ejemplo, una tercera

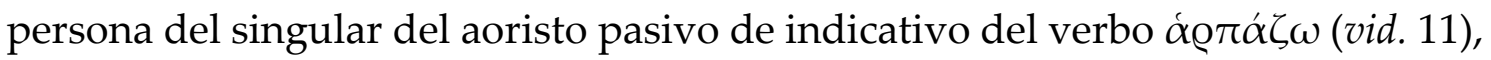
me he limitado únicamente a esta tercera persona del singular, tanto en la forma clásica como en la postclásica que denuncian. En muchas ocasiones, se encuentran ejemplos de ambas formas, la ática que recomiendan los gramáticos y la postclásica que condenan, pero solo he considerado que las formas de nueva creación se han extendido cuando superan en su uso a las formas áticas en más de un sesenta o sesenta y cinco por ciento.

Por cuestiones de extensión, en este breve estudio se muestran únicamente las conclusiones respecto a la extensión de las formas de aoristo sin profundizar qué autores prefieren las formas áticas y cuáles las de formación posterior.

\footnotetext{
${ }^{2}$ Me gustaría agradecer al Dr. José Manuel Floristán por haberme facilitado las glosas exactas en las que estos gramáticos condenan las formas no áticas de aoristo.

3 Para más información sobre los gramáticos y el aticismo, vid. Christidis (2007: 1193-1212) y Cassio (2006: 405-409).
} 


\section{AORISTOS RADICALES ATEMÁticos}

Los aoristos radicales atemáticos son bastante escasos: son los del tipo ع̋ß $\eta v$,

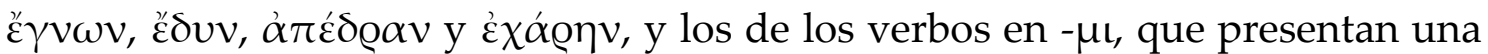
alternancia vocal larga y - $\kappa$ - en singular y vocal breve sin - $\kappa-$ en plural ( $\varepsilon \delta \omega \kappa \alpha-$ $\varepsilon ̌ \delta o \mu \varepsilon v)$. En época clásica, la -א- se extendió también al plural (cf. Willi 2018: 299, Van Emde Boas et al. 2019: 160-166), hecho que ya se observa en Heródoto: $\varepsilon \check{\delta} \omega \kappa \alpha v(1,89,4)$. La tendencia de estos aoristos en el desarrollo del griego es a desaparecer y a convertirse analógicamente en formas más comunes, como la de los aoristos sigmáticos ( Horrocks 2010: 109-110).

Dentro de este primer apartado, nos encontramos seis grupos de glosas con las raíces de los aoristos de los verbos $\delta i ́ \delta \omega \mu \mathrm{u}(1)$, en el que se extiende la - $\kappa-$ del

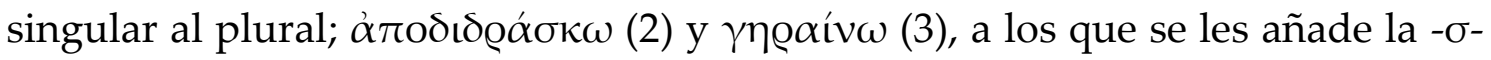

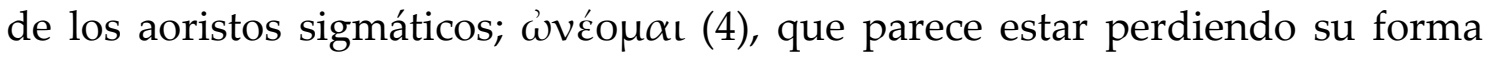

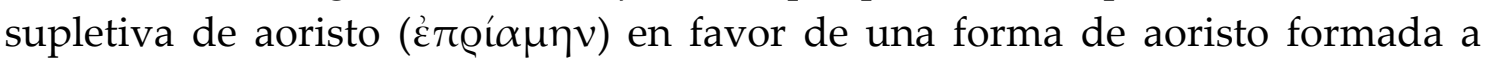
partir del tema de presente ( $\dot{\varepsilon} \omega \nu \eta \sigma \alpha ́ \mu \eta \nu) ; \varphi \theta \alpha ́ v \omega(5)$, que estaría empezando a formar su aoristo de manera sigmática, en lugar de mantener el radical atemático; y óvínuı (6), cuyo aoristo es confundido con el de óvo $\mu \alpha$ ı por el cambio de vocalismo de $\eta>\alpha$, lo que provoca el uso de óvo $\alpha$ เ ('reprochar') con el significado de óvínuı ('beneficiarse') y viceversa.

Tabla 1. Situación del aoristo radical atemático.

\begin{tabular}{|l|c|l|}
\hline \multicolumn{1}{|c|}{ Glosas } & Se ha extendido & \multicolumn{1}{|c|}{ Gramáticos } \\
\hline (1) $\dot{\alpha} \pi \varepsilon \delta \omega \kappa \kappa \alpha \mu \varepsilon v$ & Sí & Moer. $\alpha$ 19.4 \\
\hline (2) $\dot{\alpha} \pi \varepsilon ́ \delta \varrho \alpha \sigma \alpha$ & Sí & $\begin{array}{l}\text { Phryn. PS. 16, 6; Moer. } \alpha \text { 80; Hdn. } \\
\text { Philet. 423; Orus Att. A 11; Ael. Dion } \alpha \\
156 ; \text { Tim. Lex. A 977a28. }\end{array}$ \\
\hline (3) $\gamma \eta \varrho \tilde{\alpha} \sigma \alpha \iota$ & Sí & Moer. $\gamma 20$. \\
\hline (4) $\dot{\varepsilon} \omega v \eta \sigma \alpha ́ \mu \eta \nu$ & No & $\begin{array}{l}\text { Ael. Dion. } \varepsilon \text { 59; Phryn. Ecl. 108 (= 412); } \\
\text { Hdn. Philet, 410-411. }\end{array}$ \\
\hline (5) $\varphi \theta \dot{\alpha} \sigma \omega \sigma \iota v$ & Antigua & Moer. $\varphi$ 26. \\
\hline (6) $\omega v \alpha \dot{\alpha} \mu \eta \nu$ & Sí & $\begin{array}{l}\text { Phryn. Ecl. 5; Moer. } \omega \text { 5; Hdn. Philet. } \\
409 .\end{array}$ \\
\hline
\end{tabular}

En (1), (2), (3) y (6) los gramáticos condenan unas formas de aoristo que sí se han extendido en la literatura griega de los siglos I-III d.C. Concretamente, en (3) se ha impuesto por completo la forma helenística a la ática. En (4), en cambio, no se ha extendido la forma helenística que denuncian. Por último, en el grupo

${ }^{4}$ A la hora de citar se siguen las abreviaturas del Diccionario Griego-Español (DGE). 
(5), el gramático Meris se confunde al denunciar una forma de aoristo que ya utilizaban autores clásicos como Jenofonte (Cyr. 3, 2, 4: $\varphi \theta \alpha ́ \alpha \omega \mu \varepsilon v)$ o Tucídides (7, 42: $\check{\varepsilon} \varphi \theta \alpha \sigma \varepsilon v)$.

\section{AORISTOS INTRANSITIVOS EN - H- Y - $\mathrm{OH}-$}

Los aoristos en - $\eta$ - y - $\theta \eta$ - en origen eran activos, expresaban estado y tenían un sentido intransitivo ( a ello, estos aoristos adquirían un valor pasivo ( $\dot{\varepsilon} \lambda \hat{\eta} \gamma \eta v)$. De esta manera, a pesar de tener flexión activa, acabó usándose como pasivo. Por las inconveniencias de añadir el sufijo - $\eta$ - a los verbos con raíces terminadas en vocal se introdujo el sufijo $-\theta \eta-$, que adquirió sobre todo significación pasiva ( $c f$. Van Emde Boas et al. [2019: 168-179]). En los siglos I-III d.C., por un lado, hay confusión a la hora de usar uno $u$ otro sufijo $y$, por otro, los aoristos en $-\theta \eta$ - toman una $-\sigma$ -

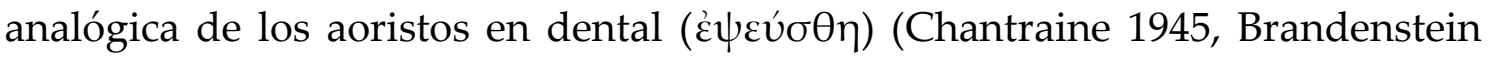
1954).

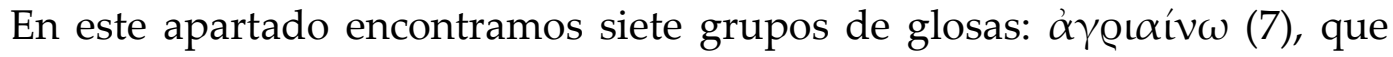
parece estar sustituyendo su aoristo supletivo ( $\dot{\alpha} \gamma \varrho \iota \omega \theta \varepsilon i ́ s)$ por uno formado a partir del tema de presente ( $\alpha \gamma \varrho \iota \alpha v \theta \varepsilon i \varsigma$ ); $\dot{\alpha} \pi \varepsilon \lambda \alpha u ́ v \omega(8)$, que presenta en algunas ocasiones una $-\sigma$ - analógica de los aoristos en dental en su aoristo pasivo; $\beta \lambda \alpha \dot{\pi} \tau \omega(9)$ y $\alpha \varrho \tau \alpha \dot{\zeta} \omega(11)$, que forman su aoristo pasivo con el sufijo - $\eta$ - en lugar

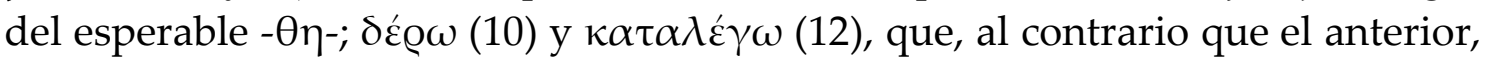
parecen formar su aoristo pasivo con $-\theta \eta$ - en lugar de con - $\eta$-. Es preciso matizar que, en los casos de $\alpha \varrho \pi \alpha ́ \zeta \omega(11)$ y $\kappa \alpha \tau \alpha \lambda \dot{\varepsilon} \gamma \omega$ (12), ambas formas de aoristo, con el sufijo - $\eta-$ y $-\theta \eta-$, están documentadas en época clásica, si bien las formas

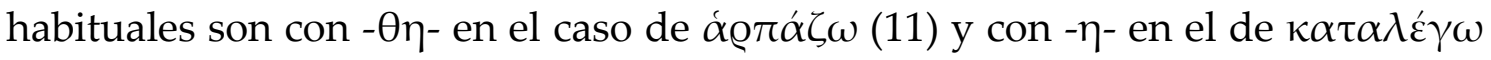
(12).

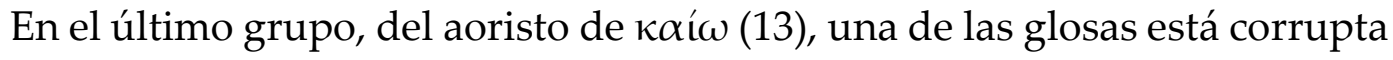
y opone una forma de aoristo ( $\kappa \alpha \tau \varepsilon \kappa \alpha u ́ \theta \eta)$ con una de futuro ( $\kappa \alpha \tau \alpha \kappa \alpha v \theta \eta ́ \sigma \varepsilon \tau \alpha \iota)$, ambas en voz pasiva. Mientras que la otra deja de manifiesto la confusión entre los sufijos - $\eta-\mathrm{y}-\theta \eta-$, que hemos visto en los grupos anteriores, por lo que en época postclásica se estaría extendiendo el aoristo con - $\eta$ - (épico) sobre el aoristo con $-\theta \eta-$. 
Tabla 2. Situación del aoristo intransitivo en - $\eta-$ y $-\theta \eta-$.

\begin{tabular}{|c|c|c|}
\hline Glosas & Se ha extendido & Gramáticos \\
\hline (7) $\alpha \gamma \gamma \varrho\llcorner\alpha v \theta \varepsilon i ́ \varsigma$ & No & Phryn. PS. 24, 12. \\
\hline (8) $\alpha \dot{\alpha} \pi \eta \lambda \alpha ́ \alpha \theta \eta$ & No & Moer. $\alpha$ 25; Moer. $\alpha 37$ \\
\hline (9) $\beta \lambda \alpha \beta \varepsilon$ cí & Sí & Antiatt. $\beta$ 14; Moer. $\beta 40$. \\
\hline (10) $\delta \alpha \varrho \varepsilon i ́ \varsigma$ & No & Antiatt. $\delta 18$. \\
\hline 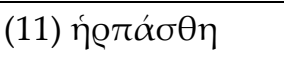 & Sí & Moer. $\eta 21$ \\
\hline (12) $\kappa \alpha \tau \alpha \lambda \varepsilon \chi \theta \varepsilon i ́ \varsigma$ & Sí & Phryn. PS 83, 1; Moer. $\kappa 7$. \\
\hline 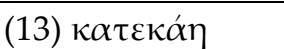 & No & Moer. $\kappa 59$ (corrupta); Antiatt. $\pi 15$. \\
\hline
\end{tabular}

Resumiendo, en tres grupos -(9), (11) y (12) - los gramáticos condenan una situación del aoristo que sí se refleja en la literatura de los siglos I-III d.C., pero en ninguno de los casos se ha impuesto la forma helenística, y en las otras cuatro - (7), (8), (10) y (13) -, en cambio, los autores utilizan más las formas áticas o, incluso, no se documenta la forma que se denuncia ( $\kappa \alpha \tau \alpha \lambda \varepsilon \chi \theta \varepsilon i ́ \varsigma$ [12]), aunque del participio pasivo del verbo $\kappa \alpha \tau \alpha \lambda \varepsilon \dot{\gamma} \omega$ con el sufijo $-\theta \eta$ - hay ejemplos de otros casos.

\section{Aoristos RAdicAles temáticos}

Los aoristos radicales temáticos no se distinguen formalmente del imperfecto. Dentro de cada verbo se distinguen del tema de presente por tener otro grado

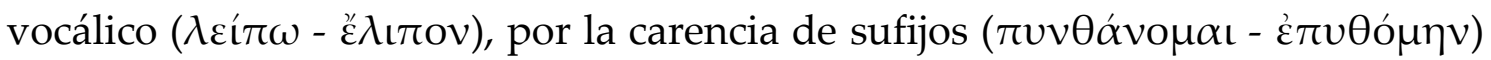
o reduplicación de presente ( $\gamma \dot{\gamma} \gamma \nu 0 \mu \alpha \iota$ - $\dot{\varepsilon} \gamma \varepsilon v o ́ \mu \eta v)$, o por tener reduplicación

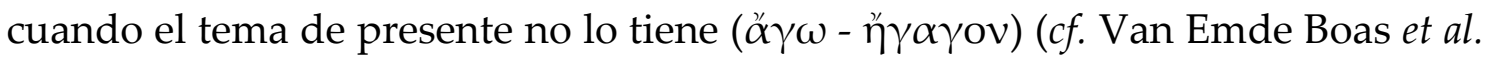
2019: 155-160). Todos ellos se mantuvieron en jónico-ático. También hay una serie de aoristos radicales en - $\alpha$ - que se alternan con aoristos radicales temáticos, como

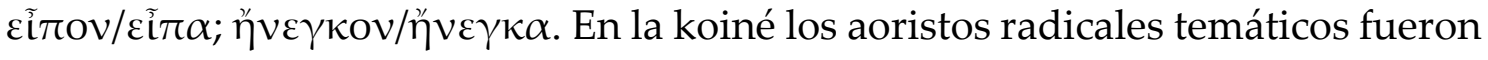
sustituidos paulatinamente por el aoristo sigmático, primero tomando las desinencias de este, como los aoristos radicales en $-\alpha-\left(\varepsilon^{\prime} \lambda \alpha \beta o v>\check{\varepsilon} \lambda \alpha \beta \alpha\right)$, y luego

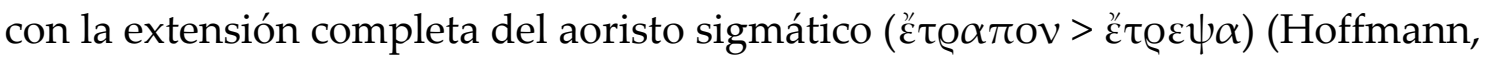
Debrunner y Scherer 1969; Dieterich 1970; Christidis 2007: 616, 697; Horrocks 2010: 143-144; Cassio 2016: 390). Antes de desaparecer por completo se crea para los aoristos radicales temáticos una terminación de tercera persona del plural

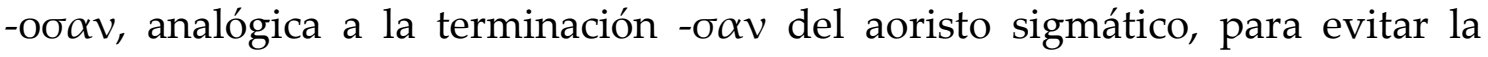
confusión con la primera persona del singular (Hoffmann, Debrunner y Scherer 1969; Dieterich 1970; Christides 2007: 988, 1442; Horrocks 2010: 321).

Dentro de este tercer grupo, los gramáticos denuncian formas de aoristo de diez verbos: $\alpha ̋ \gamma \omega(14)$, que parece estar siendo sustituido por una forma sigmática 


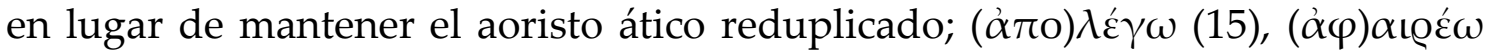

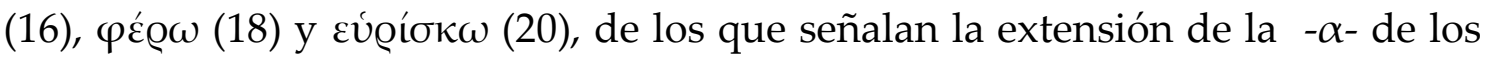

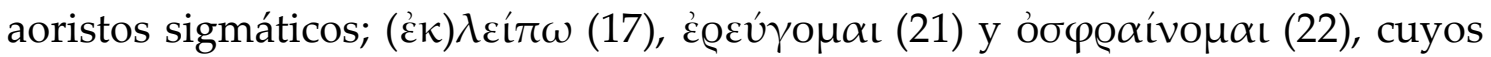
aoristos temáticos parece que empiezan a ser sustituidos por uno completamente

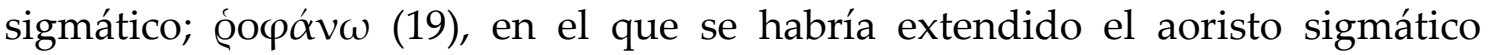

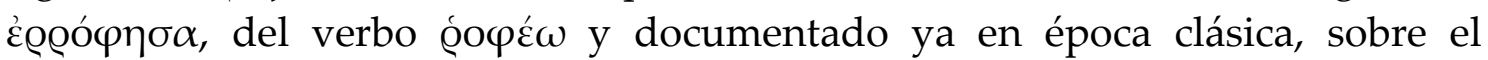
temático čo@o

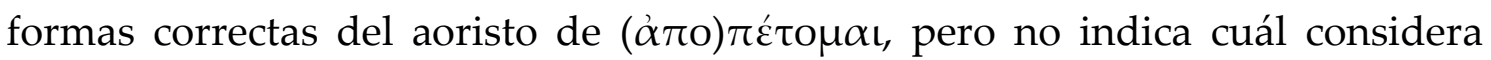
incorrecta, por lo que no se puede comparar.

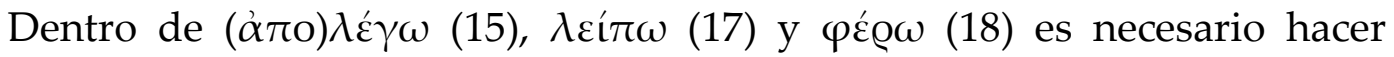

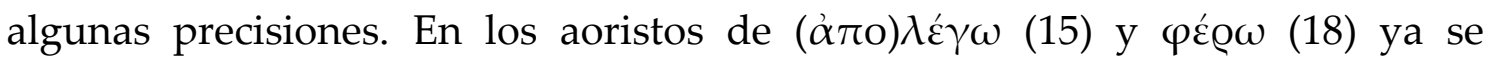

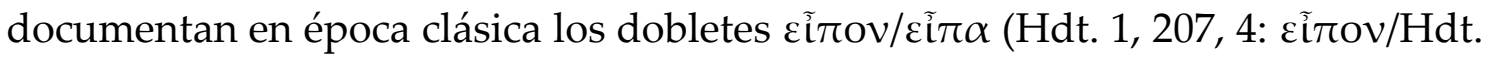

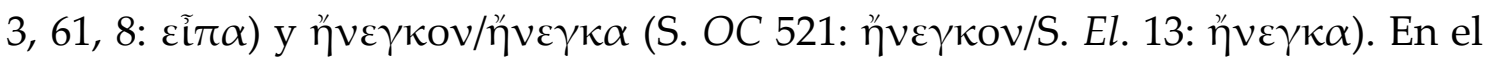
caso de ( $\dot{\varepsilon} \kappa) \lambda \varepsilon i ́ \tau \omega(17)$, el Antiaticista afirma que Aristófanes utiliza el participio $\lambda \varepsilon \dot{\imath} \psi \alpha \varsigma$ en su obra Andrómeda, pero no se nos conserva.

Tabla 3. Situación del aoristo radical temático.

\begin{tabular}{|c|c|c|}
\hline Glosas & Se ha extendido & Gramáticos \\
\hline (14) $\ddot{\alpha} \xi \omega \sigma \iota v$ & No & Phryn. Ecl. 250 (252), 326 (253) y 327 (328). \\
\hline (15) $\dot{\alpha} \pi \varepsilon i \tilde{\tau} \alpha$ & Antigua & Moer. $\alpha$ 27; Ael. Dion. $\varepsilon 22$ (156). \\
\hline (16) $\alpha \dot{\alpha} \varphi \varepsilon \dot{\lambda} \alpha \tau \tau$ & No & $\begin{array}{l}\text { Phryn. Ecl. } 154 \text { (116); Phryn. PS 288; Orus } \\
\text { Att. A 16; Hdn. De locutionum pravitatibus } \\
\text { (An. Cramer 3, 258, 16); Antiatt. } \varepsilon 31 \text {. }\end{array}$ \\
\hline 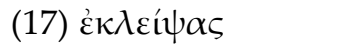 & No & Phryn. Ecl. 343; Antiatt. 106, 24. \\
\hline (18) $\dot{\varepsilon} v \varepsilon ́ \gamma \kappa \alpha \iota$ & Antigua & $\begin{array}{l}\text { Ael. Dion. } \eta 10 \text { (156); Phryn. PS. 73, 1, fr. } \\
\text { 314, fr. } 315 \text { y 63, 8; Antiatt. } \eta 1 .\end{array}$ \\
\hline (19) غ̇@@ó $\varphi \eta \sigma \alpha$ & No hay ejemplos & Ael. Dion. ع 343. \\
\hline (20) $\varepsilon u ́ \varrho \alpha \sigma \theta \alpha \iota$ & No & Phryn. Ecl. 110 (115). \\
\hline 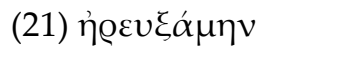 & No hay ejemplos & Phryn. PS 73, 15; Phryn. Ecl. 42. \\
\hline 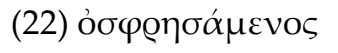 & No & Moer. o 18. \\
\hline (23) & ¿Forma incorrecta? & Phryn. PS 13, 7. \\
\hline
\end{tabular}

En tres grupos de glosas - (16), (17) y (20) - , se observa dentro del corpus que se han introducido las formas condenadas, aunque utilizan mucho más el aoristo temático. ${ }^{5}$ En cuatro grupos - (14), (19), (21) y (22) - , no se documentan

\footnotetext{
${ }^{5}$ Es necesario especificar que en [17] en la forma con preverbio no se documenta ningún ejemplo del aoristo que se condena y en la forma sin preverbio la gran mayoría de las veces que se documentan es en un único autor, Diofanto.
} 
las formas que los gramáticos denuncian, concretamente en (19) y en (21) no hay ejemplos ni de la forma helenística ni de la ática. En dos - (15) y (18) -, se confunden al denunciar formas que ya se utilizaban en época clásica. Finalmente, en una (23), es imposible saber en qué medida se refleja en los textos la situación de ese aoristo porque, como se ha mencionado antes, el gramático Frínico se limita a especificar cuáles son las formas áticas.

\section{AORISTOS SIGMÁticos}

Los aoristos sigmáticos son los que añaden a la raíz la formación - $\sigma \alpha$. Hay tres grupos de verbos que tienen un tratamiento especial al entrar en contacto con la - $\sigma-$ del aoristo con la raíz: verbos en oclusiva (gutural, labial y dental), verbos nasales y líquidos, y los verbos en vocal (cf. Van Emde Boas et al. 2019: 148-155).

Los verbos en gutural presentan una - $\xi$ - por la unión de la gutural con la

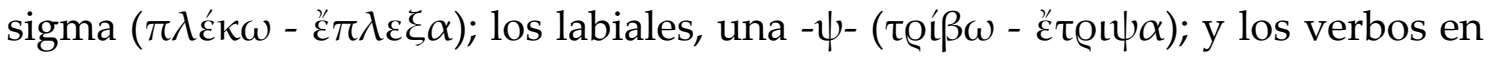
dental mantienen la - $\sigma$ - por asimilación de la dental y posterior simplificación ( $\left.\pi \varepsilon \dot{\imath} \theta \omega-\varepsilon^{\prime} \pi \varepsilon \iota \sigma \alpha\right)$. Por lo general, los verbos en - $\zeta \omega$ procedentes de una raíz con gutural presentaban un aoristo con - $\xi-(\alpha \check{\varrho} \pi \alpha \zeta \omega-\eta ̋ \varrho \pi \alpha \xi \alpha)$ y los procedentes de

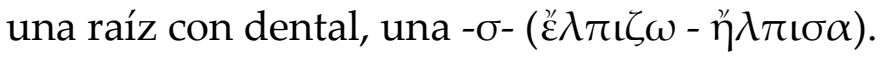

En los verbos nasales y líquidos la consonante final de la raíz $(\mu, \nu, \lambda$, @) asimila la - $\sigma$ - del sufijo de aoristo produciendo un grupo geminado que, en ático, al simplificarse en una fase posterior, produce un alargamiento compensatorio

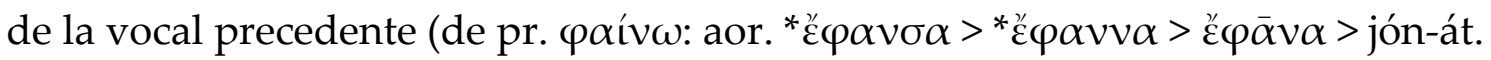

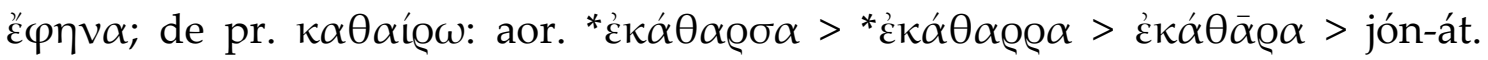

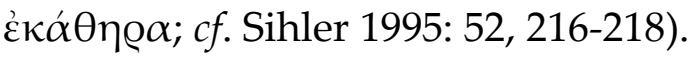

Los verbos en vocal en principio perdieron la - $\sigma$ - en posición intervocálica, pero luego la volvieron a introducir por analogía con los aoristos que la habían conservado tras consonante. Crearon sus aoristos sobre el tema alargado en $-\eta-\mathrm{u}$

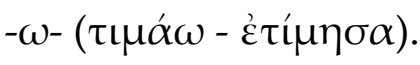

Entre estos aoristos sigmáticos, dos de los tres grupos (oclusivos y nasales y líquidos) sufrieron cambios importantes entre (o desde) el ático clásico y los siglos I-III d.C.: en los verbos en - $\zeta \omega$, ya en jónico-ático se generalizó la - $\sigma-$ y solo los verbos que tenían relación clara con la raíz gutural presente en los sustantivos mantuvieron la $-\xi-(\dot{\varepsilon} \sigma \alpha ́ \lambda \tau \iota \gamma \xi \alpha)$ (Chantraine 1945). Y los verbos en $-\pi \tau \omega$ pasaron

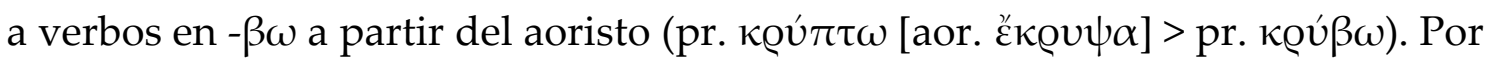
otro lado, en época de la koiné la - $\eta$ - que presentaban en el aoristo los verbos en nasal y líquida, fruto del alargamiento compensatorio, tendió a ser sustituida por la - $\alpha$ - de la raíz por analogía con el tema de presente (Dieterich 1970).

En este último apartado, nos encontramos condenas a los aoristos de once verbos: $\dot{\alpha} \lambda$ ó́ $\omega(24)$, cuyo aoristo tendría diferentes significados según fuera con - $\alpha$ - ('moler') o - $\eta$ - ('moler a palos'); $\alpha \pi о \sigma \beta \varepsilon ́ v v v \mu$ (25), que presentaría en el

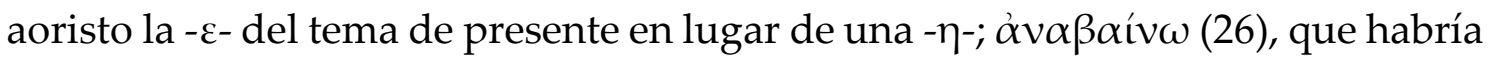
perdido la diferencia de significado entre su aoristo radical atemático ('subir') y 
su aoristo sigmático ('hacer que alguien suba') y estaría utilizando el aoristo del verbo $\beta \iota \beta \alpha ́ \zeta \omega$ con el valor causativo/factitivo; $\pi \alpha \hat{\zeta} \zeta \omega$ (27) y $\sigma \alpha \lambda \pi i ́ \zeta \omega(28)$, en los que se ve la confusión a la hora de utilizar $-\xi-$ o $-\sigma$ - para formar el aoristo;

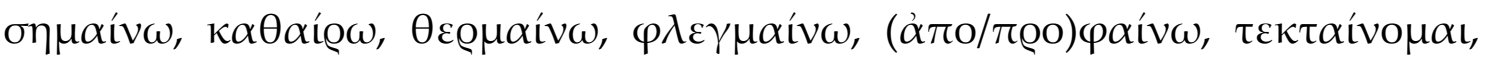

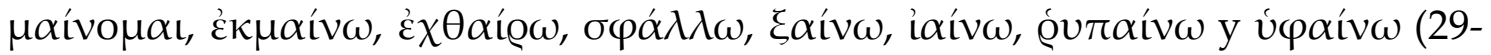
$34)$, todos ellos verbos líquidos o nasales en los que se ve, en mayor o menor medida, la extensión de la - $\alpha$ - del tema de presente al aoristo.

Tabla 4. Situación del aoristo sigmático.

\begin{tabular}{|c|c|c|}
\hline Glosas & Se ha extendido & Gramáticos \\
\hline (24) $\dot{\alpha} \lambda$ ó́ $\sigma \alpha \nu \tau \alpha$ & No hay ejemplos & Orus B 8. \\
\hline (25) $\dot{\alpha} \pi \dot{\varepsilon} \sigma \beta \varepsilon \sigma \varepsilon$ & Antigua & Phryn. PS 26, 9. \\
\hline $\begin{array}{l}(26) \dot{\alpha} v \alpha \beta \tilde{\eta} v \alpha \mathrm{\iota} / \\
\dot{\alpha} v \alpha \beta \tilde{\eta} \sigma \alpha \mathrm{\iota}\end{array}$ & No & $\begin{array}{l}\text { Phryn. PS 37, 3; Antiatt. } \kappa 63 \text {; Antiatt. } \beta \\
22 .\end{array}$ \\
\hline (27) $\pi \alpha \tilde{\imath} \xi \alpha \iota$ & Sí & $\begin{array}{l}\text { Phryn. Ecl. } 211 \text { (217); Moer. } \pi \text { 4; Moer. } \\
\text { ع } 33 .\end{array}$ \\
\hline (28) $\sigma \alpha \lambda \pi i ́ \sigma \alpha \iota$ & Sí & Phryn. Ecl. 162. \\
\hline $\begin{array}{l}\text { (29) } \sigma \eta \mu \tilde{\alpha} v \alpha \mathrm{L}, \\
\kappa \alpha \theta \tilde{\alpha} \varrho \alpha \mathrm{L}, \theta \varepsilon \varrho \mu \tilde{\alpha} v \alpha \mathrm{\iota}\end{array}$ & Sí & Phryn. Ecl. 15 (16); Phryn. PS 108, 10. \\
\hline (30) $\dot{\varepsilon} \varphi \lambda \dot{\varepsilon} \gamma \mu \alpha \nu \varepsilon$ & Sí & Phryn. Ecl. 17 (17); Orus 51. \\
\hline 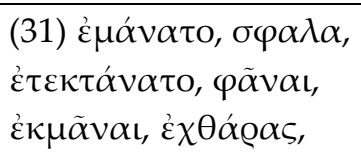 & No & Phryn. PS 108, 10. \\
\hline $\begin{array}{l}\text { (32) } \xi \tilde{\alpha} v \alpha \mathrm{L}, \mathrm{i} \tilde{\alpha} v \alpha \mathrm{l}, \\
\sigma \eta \mu \tilde{\alpha} v \alpha \mathrm{L}, \theta \varepsilon Q \mu \tilde{\alpha} v \alpha \mathrm{\iota}\end{array}$ & No & Phryn. PS fr. 341; Orus Att. B 109. \\
\hline (33) غ̇@@ú $\pi \alpha v \alpha$ & No & Moer. 126. \\
\hline (34) v́ $\varphi \alpha ́ v \alpha \sigma \alpha$ & Sí & Moer. 341. \\
\hline
\end{tabular}

En cinco grupos - (27), (28), (29), (30) y (34) - los gramáticos condenan unas formas que sí se reflejan en la literatura de estos siglos, concretamente en (27), (28) y (34) se ha impuesto por completo la forma helenística, mientras que en (29) y (30) aún se pueden ver las formas áticas. En cuatro - (24), (26), (31) y (32) - se denuncian hechos que no se observan en los textos: en (24) y (31) no se documentan ejemplos de las formas condenadas; en (32) solo dos de los cuatro

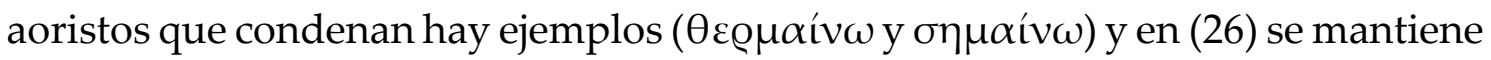
la diferencia de significado factitivo o no según sea sigmático o no. Por último, en el grupo (25) Frínico denuncia una forma que ya se utilizaba en época clásica. 


\section{CONCLUSIONES}

Después de haber analizado brevemente lo que sucede en los treinta y cuatro grupos de glosas, podemos observar que los cambios más habituales en los aoristos radicales atemáticos (1-6) son la regularización interna de los

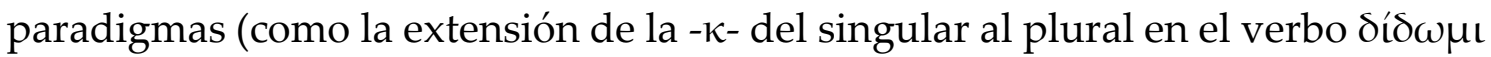
[1] y la regularización del aoristo mediante la extensión de la - $\sigma$ - de los aoristos sigmáticos).

En los aoristos intransitivos en $-\eta-y-\theta \eta-(7-13)$, por su parte, nos encontramos la introducción de una - $\sigma$ - analógica de los aoristos en dental en su aoristo pasivo (8) y la confusión entre ambos sufijos, de tal manera que algunos verbos en los que sería esperable la utilización de - $\eta$ - toman en su lugar $-\theta \eta$ - y viceversa -(9) o (10), por ejemplo.

Respecto a los aoristos radicales temáticos, vemos una paulatina regularización hacia los aoristos sigmáticos mediante la introducción, en un primer lugar, de la $-\alpha$ - propia de estos aoristos - (14) o (20), por ejemplo- y, después, de la - $\sigma--(17)$ o (22), por ejemplo. Aun así, la tendencia más habitual de los autores de los siglos I-III d.C. es la utilización de las formas temáticas antes que las sigmáticas.

Por último, en los aoristos sigmáticos, los errores más habituales respecto al ático del siglo V a.C. son la confusión entre el uso de $-\sigma-\mathrm{o}-\xi-$ en los verbos en $-\zeta \omega-(27)$ y (28) - y la extensión de una - $\alpha$ - por analogía con el tema de presente en detrimento de la $-\eta$ - fruto del alargamiento compensatorio en los verbos en nasal y líquida (29-34).

En lo que concierne a si los gramáticos aticistas denuncian unas formas de aoristo que se reflejan en la literatura de los siglos I-III d.C., se puede observar que en dieciocho grupos de glosas, catorce - (4), (7), (10), (13), (14), (16), (17), (20), (22), (26), (31), (32), (33) - más los cuatro en las que ambas formas son áticas -(5), (15), (18), (25) - , de los treinta y cuatro grupos - lo que supone más de la mitad de las glosas - los gramáticos denuncian una situación del aoristo que no se refleja en el corpus analizado, ya sea porque la forma postclásica, a pesar de estar presente, no se utiliza tanto como la ática, ya sea porque condenan formas de aoristo que ya están presentes en el ático clásico.

Por otro lado, en doce de los treinta cuatro grupos de glosas -(1), (2), (3), (6), (9), (11), (12), (27), (28), (29), (30), (34) - , los gramáticos condenan aoristos que sí se han extendido notoriamente por la literatura de estos siglos. Y, en cuatro grupos -(19), (21), (23), (24) - , no puede saberse porque no se documenta ningún ejemplo o porque no se especifica cuál es la forma helenística que se condena (23). 
Todo ello queda resumido en el siguiente gráfico:

Figura 1. Extensión de los aoristos denunciados.

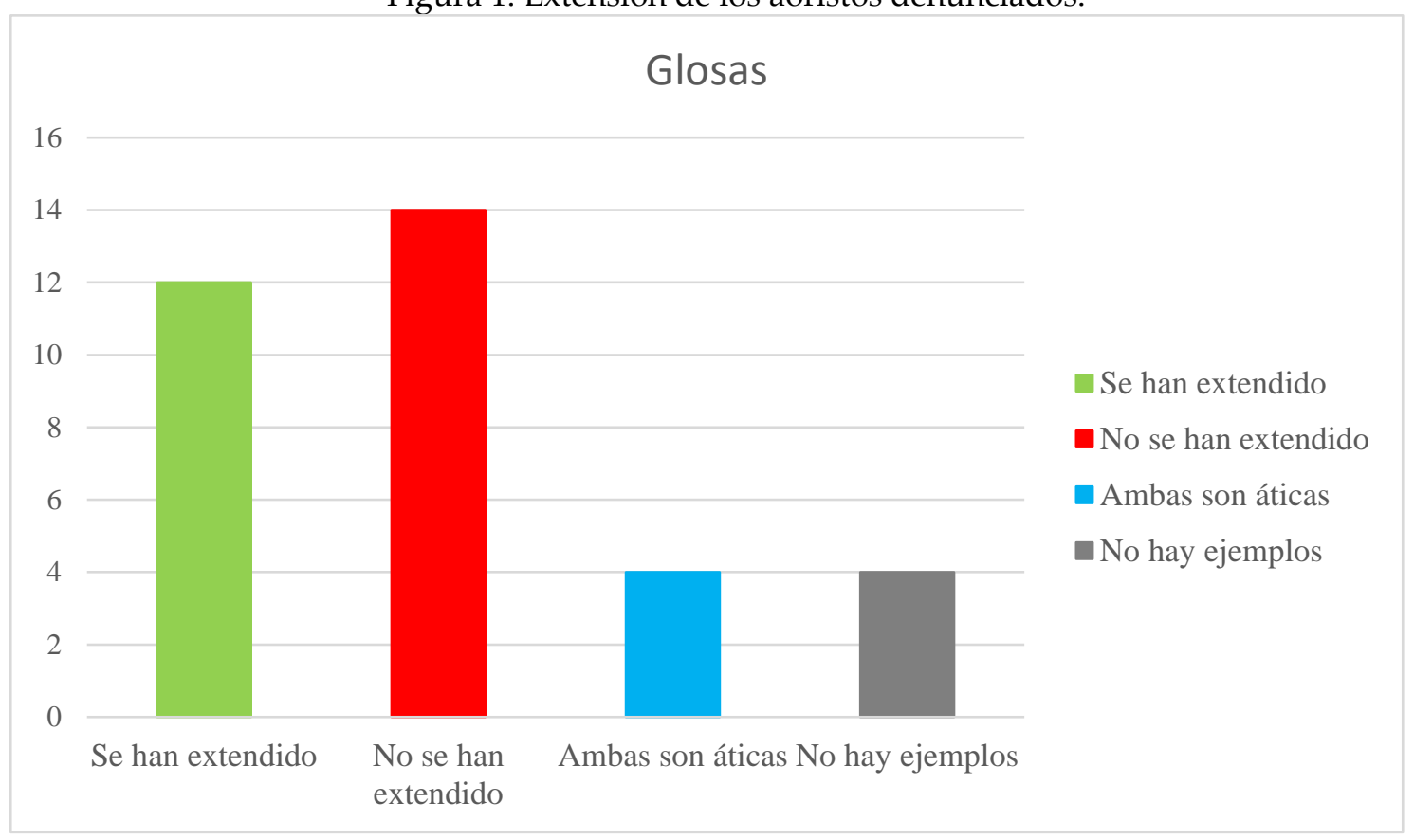

Por último, es necesario precisar que, para interpretar adecuadamente estos datos, hay que tener en cuenta que se refieren únicamente a los testimonios literarios y no se ha de cometer el error de asociarlos automáticamente al griego hablado del momento. Durante el uso de la koiné, la lengua hablada se fue separando cada vez más de la escrita, que tendió a parecerse lo más posible al dialecto ático de época clásica. Como consecuencia, desde principios de época bizantina, o incluso antes, se estableció una diglosia entre la lengua vernácula, que evolucionaba paulatinamente hacia el griego moderno, y la lengua literaria, más conservadora por elitismo frente a lo popular y lo «bárbaro».

Según el grado de conocimiento del griego del autor, el público al que estuviera dirigido el escrito y la claridad o expresividad que se buscase, existen diferentes variedades de lengua escrita, algunas con más elementos de la lengua hablada que otras. Por lo que, en ocasiones, si se trata de un texto de algún autor culto, como podría ser Plutarco, no siempre se pueden observar los cambios más extendidos y presentes en la lengua hablada (Egea 1988: 26-29; Horroks 2010: 135, 229-230).

\section{BIBLIOGRAFÍA}

BRANDENSTEIN, W. (1954), Griechische sprachwissenschaft i: einleitung, lautsystem, etymologie, Berlín, De Gruyter. (trad. de García Yebra, V., Lingüística griega, Madrid, Gredos, 1964)

CAssio, A. C. (ed.) (2016²), Storia delle lingue letterarie greche, Florencia, Le Monnier Università. 
Chantraine, P. (1945), Morphologie historique du grec, París, Klincksieck. (trad. de Sanz Franco, F., Morfología histórica del griego, Barcelona, Avesta, 1983)

CHRISTIDIS, A. F. (ed.) (2007), A History of Ancient Greek. From the Beginnings to Late Antiquity, Cambridge, Cambridge University Press.

DIETERICH, K. (1970), Untersuchungen zur Geschichte der griechischen Sprache von der hellenistischen Zeit bis zum 10 Jahrhundert n. Chr, reimpr., Hildesheim, Zúrich y Nueva York, OLMS.

EGEA, J. M (1988), Gramática de la Crónica de Morea: un estudio sobre el griego medieval, Vitoria, Universidad del País Vasco.

HOFFMANN, O., DEBRUNNER, A. y SCHERER, A. (1969), Geschichte der griechischen Sprache, Berlín, De Gruyter. (trad. de Moralejo Laso, A., Historia de la lengua griega, Madrid, Gredos, 1986)

HORROCKS, G. $\left(2010^{2}\right)$, Greek: A history of the language and its speakers, Chichester, WileyBlackwell.

SIHLER, A. (1995), New Comparative Grammar of Greek and Latin, Oxford, Oxford University Press.

VAN EMDE BOAS, E. et alii (2019), The Cambridge Grammar of Classical Greek, Cambridge, Cambridge University Press.

WILLI, A. (2018), Origins of the Greek Verb, Cambridge, Cambridge University Press. 
